\title{
Microsatellite in Coriander: A Cross Species Amplification within Apiaceae Family
}

\author{
Sharda Choudhary ${ }^{1}$, Geetika Jethra ${ }^{2}$, Radheshyam Sharma ${ }^{1}$ and Arvind Kumar Verma ${ }^{1}$ \\ ${ }^{1}$ ICAR-National Research Centre on Seed Spices, Ajmer, Rajasthan-305 206, India \\ ${ }^{2}$ Banasthali Vidyapith, P.O. Banasthali Vidyapith, Rajasthan-304022, India \\ *Corresponding author
}

\begin{tabular}{l} 
Ke y w o r d s \\
Apiaceae, \\
Coriandrum \\
sativum, Cross- \\
Transferability, \\
Microsatellite, SSRs \\
\hline Article Info \\
$\begin{array}{l}\text { Accepted: } \\
26 \text { April } 2017 \\
\text { Available Online: } \\
10 \text { May } 2017\end{array}$ \\
\hline
\end{tabular}

\section{Introduction}

Coriander (Coriandrum sativum $\mathrm{L}$ ) is one of the important seed spice crops after cumin. The crop belongs to the family Apiaceae and have diploid chromosome number $2 \mathrm{n}=22$. The coriander seeds and foliage possess a unique fragrance and taste, which are used all over the world as culinary spice, flavouring agent. In addition to its use as seed spice in our routine cooking, recent studies have shown its pharmaceutical and medicinal importance (Aruna and Baskaran, 2010). Coriander is native to the Mediterranean and commercially produced in India, Russia, Romania, Ukraine,
Morocco, Canada, France, Central America, Mexico, and USA (Qureshi et al., 2009). India is the largest producer and accounting for 5.5 lakh tonnes per annum of seeds from 5.8 lakh ha area. The quantity of foliage production and consumption remains unaccounted. India is also the highest consumer and exporter of coriander. In India, Rajasthan, Gujarat, Andhra Pradesh, Uttar Pradesh, Madhya Pradesh, Himachal Pradesh are the major coriander growing states in India. Very limited molecular and genomic studies on it, hinders the molecular research. 
In addition, a lack of coriander molecular markers limits the process of molecular genetics breeding. Till date very limited literature is available for molecular studies in coriander. Molecular genetic breeding is also very difficult in coriander as lack of molecular markers limits the process. Problems associated with conventional breeding can be overcome by the use of molecular marker assisted selection. However, only a few molecular markers have been reported in coriander like RAPD (AlKordy et al., 2013; Singh et al., 2013). A very popular and informative marker SSR is still lacking for coriander crop. Development of new SSRs for new species like coriander is very time consuming, expensive and requires isolation, cloning, sequencing and characterization of microsatellite loci. Available collections of ESTs (Expressed Sequence Tags) on public domain can be explored for microsatellite development (Kantety et al., 2002). This approach is, only applicable to well-studied species where abundant molecular data is available. Another very economic and reliable approach for the genome with very limited DNA sequence information is screening of the conservation of microsatellite flanking regions in closely related Species (Bushar et al., 2001; Schnell et al., 2005). Primer screening from different relevant sources are the most potential chance for development of SSR marker for species without prior sequence information (Barbara et al., 2007). Transferability of microsatellite markers within genus, across the species (Ciprian et al., 1999; Butcher et al., 2000) and in leguminaceae across genera transferability, belongs to same family has been reported (Peakall et al., 1998), Fagaceae (Al-drich et al., 2003) and Casuarinaceae (Ramasamy et al., 2005). In the present study, we analyzed the transferability of 100 microsatellite loci originally belongs to carrot (daucus carota) as both the crops coriander and carrot belongs to the same family.
Microsatellites (SSRs) have been widely recognized as the powerful marker of choice for plants and animals which is considered as the very informative genetic marker. Microsatellites normally occur in non-coding regions of the genome but they can also occur coding regions or in either $3^{\prime}$ or 5' UTRs of ESTs. Microsatellites (SSRs) are composed of short (2-5 base pairs), tandemly repetitive tracts of DNA in which certain DNA motifs are repeated, typically 5-50 times (Caporale $e t$ al., 2003). For example, the sequence TATATATATA is a dinucleotide SSR, and GTCGTCGTCGTCGTC is a trinucleotide SSR (with A being Adenine, T Thymine, G Guanine, and C Cytosine). Repeat DNA motifs of four and five nucleotides are referred to as tetra- and pentanucleotide SSRs, respectively. This marker is abundant, codominant, hypervariable and transportable between species and are distributed throughout the animals and plants genome (Roder et al., 1995; Gupta and Varshney, 2000; Jarne and Lagoda, 1996). Due to their high polymorphism, and easy use with the PCR, microsatellite loci are considered as ideal markers (Kuleung et al., 2004, Peakall et al., 1998). In plants, SSRs are useful for cultivar identification, genetic linkage mapping and marker assisted selection in breeding and population genetic studies (Barreneche et al., 1998; Butcher et al., 2000; Steinkellner et al., 1997).

\section{Materials and Methods}

\section{Plant material and DNA extraction}

To test the cross-species transferability of microsatellite loci, we obtained five samples of coriander Variety ACr-1 from Gene Bank, ICAR-National Research Centre on Seed Spices, Tabiji, Ajmer (Rajasthan), India. DNA was extracted from frozen leaves of 20 days old seedlings using cetyltrimethyl ammonium bromide (CTAB) method 
described by Doyle and Doyle (1990). The quality and quantity of DNA was determined by electrophoresis on $0.8 \%$ agarose gel. DNA samples were diluted to $20 \mathrm{ng} / \mu \mathrm{l}$ for polymerase chain reaction (PCR) amplification. A total of hundred primers screened for cross-species amplification were synthesized by Xcelris genomics (India).

\section{PCR amplification with microsatellites and data analysis}

Hundred microsatellite loci previously developed for carrot by Cavagnaro et al., (2011) were tested for cross amplification in coriander species. PCR reactions $(20 \mu \mathrm{l})$ were conducted in $\mathrm{C} 1000^{\mathrm{TM}}$ Thermal cycler (BIORAD) containing $12.8 \mu \mathrm{l}$ water, $2 \mu \mathrm{l} 10 \mathrm{X}$ DNA polymerase buffer, $2 \mu \mathrm{l}$ dNTPs $(2 \mathrm{mM}$ each), $0.5 \mu \mathrm{l}$ of each primer at $100 \mu \mathrm{M}, 0.2 \mu \mathrm{l}$ Taq DNA Polymerase at $5 \mathrm{u} / \mu \mathrm{l}$ (SRL, biolit $^{\mathrm{TM}}$ ) and $2 \mu \mathrm{l}$ genomic DNA. Thermal cycling conditions were as follows: initial denaturation at $94^{\circ} \mathrm{C}(4 \mathrm{~min})$, followed by 40 cycle of appropriate primer annealing temperature $(1.0 \mathrm{~min})$, elongation at $72^{\circ} \mathrm{C}(1.0$ min), and denaturation at $94^{\circ} \mathrm{C}(20 \mathrm{sec})$. A final elongation step was allowed for 10.0 $\min$ at $72^{\circ} \mathrm{C}$.

Electrophoresis was carried out for 3 hours at $150 \mathrm{~V}$ on $1.5 \%$ high-resolution agarose. For each $100 \mathrm{ml}$ of TAE, TAE gels were supplemented with 5 ul $(5.0 \mathrm{mg} / \mathrm{ml})$ of ethidium bromide. For each microsatellite locus, sizes of the allele were estimated by comparison with standard DNA marker (100bp, SRL Biolit ${ }^{\mathrm{TM}}$ ) and were scored across all the samples manually. Amplification of products was scored for sharp and reproducible bands only.

\section{Results and Discussion}

To evaluate the potential utilization of SSRs, two different types of microsatellites were used for transferring carrot SSR markers to coriander viz; genomic microsatellites (GSSRs) and BAC end sequences (BSSRs) based SSRs (Cavagnaro et al., 2011) were tested in five sample of coriander. Specific PCR amplification were considered for those amplifications which were of approximate length expected for that particular SSR in carrot and those with different size were considered as non specific amplification.

Pattern of cross species amplification in plant across genera is beginning to emerge as a source for developing microsatellites in lesser-studied or orphan crops. Very few studies are available for transferability of microsatellites to related species.

In present study cross-species amplification was scored as positive only when sharp band/bands were reproduced in at least two out of three PCR replicates. Using this criterion Under standard, high-stringency PCR amplification conditions, out of the hundred microsatellite loci assayed, 39\% were amplified in at least one of the used coriander samples (Table: 1). The remainder could not be consistently scored or failed to amplify.

When hundred microsatellite loci of carrot were amplified in PCR for cross-species transferability at annealing temperature $54^{\circ} \mathrm{C}$ or below, very feint and stutter bands were produced and therefore the annealing temperature was increased to $55^{\circ} \mathrm{C}$ and up to $65^{\circ} \mathrm{C}$ for few primer pairs (Table: 1). Such modification in the PCR amplification protocol specially primer annealing temperature are recommended for the successful transfer of microsatellite loci between species (Rosotto et al., 2001).

Out of tested 100 carrot SSRs only 39 were showing PCR amplification with coriander, the putative SSR loci of 35 primers were monomorphic. 
Table.1 Characteristics of 39 carrot microsatellites showing amplification in coriander

\begin{tabular}{|c|c|c|c|c|c|c|c|c|c|}
\hline S.N. & Microsatellite & SSR Motif & Sequence 5'-3 & $\begin{array}{l}\text { Annealing } \\
\text { temp }{ }^{\circ} \mathrm{C}\end{array}$ & $\begin{array}{l}\text { Amplicon } \\
\text { size (bp) }\end{array}$ & $\begin{array}{l}\text { NCBI } \\
\text { number }\end{array}$ & $\begin{array}{l}\text { GC } \\
\text { content }\end{array}$ & $\begin{array}{l}\text { Allel } \\
\text { Size } \\
\text { (bp) }\end{array}$ & Spec \\
\hline 1 & GSSR-4 & $\begin{array}{l}\text { (TCTA)21 } \\
\text { (p4) }\end{array}$ & $\begin{array}{l}\text { F: CAATCTTGCCACTAAAAGAGCA } \\
\text { R: CAGATACAATAGACAGGAAACATCG }\end{array}$ & $55 / 55$ & 314 & FJ816114 & 40.9 & 350 & + \\
\hline 2 & GSSR-5 & $\begin{array}{l}(\mathrm{AC}) 9 \\
(\mathrm{AC}) 6(\mathrm{p} 2)\end{array}$ & $\begin{array}{l}\text { F: ATAATAAACCCAACCAGACCCC } \\
\text { R: ATCAGGCAAATCCCATACTGAC }\end{array}$ & $54 / 58$ & 120 & FJ816115 & 45.5 & 120 & + \\
\hline 3 & GSSR-10 & $(\mathrm{GACA}) 7(\mathrm{p} 4)$ & $\begin{array}{l}\text { F: CTTAGTAGTAGCACACACCAGACG } \\
\text { R: GAGCTGAACGAGTCAGAAAGG }\end{array}$ & $55 / 55$ & 255 & FJ816120 & 50 & 250 & + \\
\hline 4 & GSSR-16 & (TG)9tacgc(ATGT)3 (c) & $\begin{array}{l}\text { F: ATGCAAACGACAATATCCACAG } \\
\text { R: GCCCAGCCACTTCCTAGAT }\end{array}$ & $57 / 57$ & 212 & FJ816126 & 40.9 & 220 & + \\
\hline 5 & GSSR-35 & $(\mathrm{GA}) 13(\mathrm{p} 2)$ & $\begin{array}{l}\text { F: AATTCACAATCACCGACTCTCC } \\
\text { R: ACGTCAAAGCTCCTGTTCATTT }\end{array}$ & $55 / 57$ & 173 & FJ816145 & 45.5 & 175 & + \\
\hline 6 & GSSR-37 & $\begin{array}{l}\text { (TATG)7 } \\
\text { (TTGC)3 (p4) }\end{array}$ & $\begin{array}{l}\text { F: CGAGGGAGAATGACGAAAATTA } \\
\text { R: TCTGTGACGAGTAGGATCAGGA }\end{array}$ & $55 / 55$ & 197 & FJ816147 & 40.9 & 200 & + \\
\hline 7 & GSSR-40 & $(\mathrm{CAT}) 7(\mathrm{p} 3)$ & $\begin{array}{l}\text { F: TAGAAGCTCCAACAAATCACCC } \\
\text { R: CAAGGAACCCTAGATCACAAATG }\end{array}$ & $55 / 55$ & 171 & FJ816150 & 45.5 & 900 & - \\
\hline 8 & GSSR-43 & (TG)12 (p2) & $\begin{array}{l}\text { F: TTCTTCACCTATGTTGGGGC } \\
\text { R: CGTTCATATGCACAACACTCA }\end{array}$ & $58 / 55$ & 198 & FJ816152 & 50 & $\begin{array}{l}200 \\
700\end{array}$ & $\mathrm{P}$ \\
\hline 9 & GSSR-81 & $\begin{array}{l}(\mathrm{CT}) 15 \\
(\mathrm{CT}) 8(\mathrm{p} 2)\end{array}$ & $\begin{array}{l}\text { F: TGGGTCTCTGGCCAATTCTA } \\
\text { R: CAATTTGCACATAACTCATCAAG }\end{array}$ & $58 / 58$ & 228 & FJ816190 & 50 & 900 & - \\
\hline 10 & GSSR-87 & $\begin{array}{l}\text { (TCT) } 15 \\
\text { (TCT) } 4(\mathrm{p} 3)\end{array}$ & $\begin{array}{l}\text { F: CCAACAACCATCCAACAAACTA } \\
\text { R: AGTCGTCCGATAAGCGAATCTA }\end{array}$ & $58 / 58$ & 391 & FJ816196 & 40.9 & 400 & + \\
\hline 11 & GSSR-92 & (ACAT)11 (p4) & $\begin{array}{l}\text { F: AGAAGGCATCGTGTGTTCATAA } \\
\text { R: CAACGGTGATTAAGTGGGTTCT }\end{array}$ & $55 / 55$ & 276 & FJ816201 & 40.9 & 250 & + \\
\hline 12 & GSSR-96 & $(\mathrm{CAT}) 5(\mathrm{p} 3)$ & $\begin{array}{l}\text { F: AGCGTCGTTTTCGCGAGT } \\
\text { R: CGCGGTTAAAGCAAAGCTAAT }\end{array}$ & $55 / 60$ & 334 & FJ816205 & 55.6 & $\begin{array}{l}350 \\
400 \\
600 \\
700\end{array}$ & $\mathrm{P}$ \\
\hline 13 & GSSR-97 & $\begin{array}{l}(\mathrm{GA}) 8 \quad(\mathrm{GA}) 7 \\
(\mathrm{AG}) 7 \text { aagtattcca(AG)6 (c) }\end{array}$ & $\begin{array}{l}\text { F: GGCAAAGAAACAGATTTGGAGA } \\
\text { R: CTGCCCTAGCATCAAAACAAAC }\end{array}$ & $55 / 55$ & 265 & FJ816206 & 40.9 & 300 & + \\
\hline 14 & GSSR-100 & $(\mathrm{ACA}) 4(\mathrm{p} 3)$ & $\begin{array}{l}\text { F: TTCCCCTTGTCGTAACTCCA } \\
\text { R: CTGCAACTCTGGATTTGAGGT }\end{array}$ & $54 / 55$ & 362 & FJ816209 & 50 & 350 & + \\
\hline 15 & GSSR-101 & $\begin{array}{l}(\mathrm{GA}) 6 \\
(\mathrm{GA}) 10(\mathrm{p} 2)\end{array}$ & $\begin{array}{l}\text { F: TGGGGTGGTTTTGTCGTATT } \\
\text { R: TGGGGTGGTTTTGTCGTATT }\end{array}$ & $54 / 58$ & 328 & FJ816210 & 45 & 350 & + \\
\hline 16 & GSSR-104 & $(\mathrm{GTT}) 5(\mathrm{GAG}) 4(\mathrm{c})$ & $\begin{array}{l}\text { F: TGGTTCTGAGGAAGATGTTTGA } \\
\text { R: GCCTGCACTAAATTGATGACAC }\end{array}$ & $54 / 60$ & 330 & FJ816213 & 40.9 & 350 & + \\
\hline 17 & GSSR-107 & $\begin{array}{l}(\mathrm{ATAC}) 8 \\
(\mathrm{ACAT}) 4(\mathrm{p} 4)\end{array}$ & $\begin{array}{l}\text { F: TTCTGGTCTTTTGACATGAAGG } \\
\text { R: CGGATTTGAGGTGAGTTGAATA }\end{array}$ & $54 / 55$ & 265 & FJ816216 & 40.9 & 300 & + \\
\hline 18 & GSSR-111 & (ATAC)3atccatc(CATA)9tat(CA)20 (c) & F: GAGGAAGGGTAGATCCAGTCA & $55 / / 55$ & 355 & FJ816220 & 52.4 & 350 & + \\
\hline
\end{tabular}




\begin{tabular}{|c|c|c|c|c|c|c|c|c|c|}
\hline 19 & GSSR-112 & (CT)12(CA)6a(AC)6 (c) & $\begin{array}{l}\text { R: ATGGGATGTCTTTCCCCTCTAT } \\
\text { F: TCTTGTYYAAGAAMACCACA } \\
\text { R. }\end{array}$ & $55 / 55$ & 197 & FJ816221 & 37.5 & 200 & + \\
\hline 20 & GSSR-113 & (TTG)4 & $\begin{array}{l}\text { R: GICCACACCAAGIAIGC IC } \\
\text { F: AGTGGTTGTGAGGTGATTGTG }\end{array}$ & $55 / 55$ & 336 & FJ816222 & 45.5 & 600 & $-\mathrm{P}$ \\
\hline & & (TGT)4 (p3) & R: TATGTCGGAAAGGTTCAATGCT & & & & & 900 & \\
\hline 21 & GSSR-131 & (GAT)11 (p3) & $\begin{array}{l}\text { F: AAATTACTGGAGATGGAGCGAG } \\
\text { R: GTTTGTTGATTCGGACTTTGTG }\end{array}$ & $55 / 55$ & 165 & FJ816240 & 45.5 & 150 & + \\
\hline 22 & GSSR-132 & (TGT)4 (p3) & $\begin{array}{l}\text { F: CAGGTAGTGGTTGWGAGYTTGA } \\
\text { R: TCCCCTTGTCGTAACTCCATAC }\end{array}$ & $54 / 56$ & 332 & FJ816241 & 47.7 & 350 & + \\
\hline 23 & GSSR-136 & (TG)5 (p2) & $\begin{array}{l}\text { F: GAAGAAAGGAGCTAAAGTGGGA } \\
\text { R: CCCATTCCATTTTCACTTGC }\end{array}$ & $58 / 58$ & 426 & FJ816244 & 45.5 & 450 & + \\
\hline 24 & GSSR-138 & $\begin{array}{l}\text { (GT)5ata(GT)7 } \\
\text { (AG)21 (p2) }\end{array}$ & $\begin{array}{l}\text { F: CGCTCGAGTTTCGTAGAGT } \\
\text { R: CCTCCCCAACTCAATCCAAT }\end{array}$ & $56 / 56$ & 519 & FJ816246 & 52.6 & 500 & + \\
\hline 25 & GSSR-139 & (TGTC)4 (p4) & $\begin{array}{l}\text { F: GCAAGTGTTTCGTGACATGC } \\
\text { R: AACATGAGTTAATCGAAGGGGA }\end{array}$ & $56 / 56$ & 365 & FJ816247 & 50 & 350 & + \\
\hline 26 & GSSR-140 & $(\mathrm{TC}) 10(\mathrm{p} 2)$ & $\begin{array}{l}\text { F: GGATACGAAGGAAAGACTCCAC } \\
\text { R: AGGAGAGTAAAAGATTGAGGACTTG }\end{array}$ & 5658 & 158 & FJ816248 & 50 & 700 & - \\
\hline 27 & GSSR-141 & (CT) $8 \operatorname{cct}(\mathrm{CCTC}) 3(\mathrm{c})$ & $\begin{array}{l}\text { F: CTACAATACACCAACATACCAAAGG } \\
\text { R: GGCTACAATGAACCAAGAAATG }\end{array}$ & $55 / 55$ & 184 & FJ816249 & 40 & 700 & - \\
\hline 28 & GSSR-142 & $(\mathrm{AAAC}) 5(\mathrm{p} 4)$ & $\begin{array}{l}\text { F: GTAGAAACCTTTTGGCAGTAACG } \\
\text { R: CCAAGACCATGAAGAAATCACTC }\end{array}$ & $56 / 56$ & 138 & FJ816250 & 43.5 & 150 & + \\
\hline 29 & GSSR-143 & (TATC)4(TA)7 (c) & $\begin{array}{l}\text { F: GGGAAGAACTAAAACCAACACA } \\
\text { R: TCAGTAAATCAGGAGTGCAGAA }\end{array}$ & $55 / 55$ & 346 & FJ816251 & 40.9 & 350 & + \\
\hline 30 & GSSR-149 & $\begin{array}{l}(\mathrm{GAGAGG}) 3(\mathrm{GA}) 8 \quad(\mathrm{c}) \\
(\mathrm{p} 2)\end{array}$ & $\begin{array}{l}\text { F: TGAAGCAACTCGTGATACAGAGA } \\
\text { R: TTCTCTTGTCCTGGTTAGCTC }\end{array}$ & $57 / 57$ & 342 & FJ816257 & 43.5 & 350 & + \\
\hline 31 & GSSR-153 & $\begin{array}{l}\text { (TC)15 (p2) } \\
\text { (TAGTTG)2 (p6) }\end{array}$ & $\begin{array}{l}\text { F: GTCACCCCACCAAGAAAAA } \\
\text { R: CCAAACACAAGACAGTCAAATG }\end{array}$ & $56 / 56$ & 255 & FJ816261 & 47.4 & 500 & - \\
\hline 32 & GSSR-154 & (TC)11 (p2) & $\begin{array}{l}\text { F: CTTATATGTGATGGCGTCGAAA } \\
\text { R: GACTGCACCGCTCCTAACTC }\end{array}$ & $56 / 56$ & 328 & FJ816262 & 40.9 & 350 & + \\
\hline 33 & GSSR-18 & $\begin{array}{l}(\mathrm{TG}) 7 \mathrm{tt}(\mathrm{TG}) 4(\mathrm{c}) \\
(\mathrm{TG}) 7(\mathrm{P} 2)\end{array}$ & $\begin{array}{l}\text { F: TTGGGGAACAAGCAACTCAG } \\
\text { R: CTCTCTTGAATGTTGGCATACAC }\end{array}$ & $54 / 55$ & 130 & FJ816126 & 50 & 150 & + \\
\hline 34 & GSSR-58 & $\begin{array}{l}\text { (TAAAA)2 } \\
\text { (CAAAA)2 (p5) }\end{array}$ & $\begin{array}{l}\text { F: CAAGAACTACGCACATAACTCA } \\
\text { R: TGCTCTAGCCTTTTTACCGC }\end{array}$ & $54,56 / 55$ & 301 & FJ816167 & 40.91 & 300 & + \\
\hline 35 & GSSR-61 & (CTCA)4 (p4) & $\begin{array}{l}\text { F: TGTATTAAAGGCACAACTGGA } \\
\text { R: CCTTGTCACCTAGAGTGG }\end{array}$ & $54 / 65$ & 402 & FJ816170 & 38.1 & 400 & + \\
\hline 36 & BSSR-1 & (TCT)4 (p3) & $\begin{array}{l}\text { F: TTCTTGGTCTGTTGATGTCAGTGTAG } \\
\text { R: TGGATATAGAAGCCATCAGACTTGAG }\end{array}$ & $56 / 56$ & 180 & FJ147759 & 42.3 & 150 & + \\
\hline 37 & BSSR-6 & (TAAT)3 (p4) & $\begin{array}{l}\text { F: GTCGCGGAAGTGAATCTGA } \\
\text { R: TGTTCGTAACTTGCTTGATCG }\end{array}$ & $54 / 60$ & 223 & FJ147807 & 52.6 & 600 & - \\
\hline 38 & BSSR-8 & (AT)6 (p2) & $\begin{array}{l}\text { F: TGAAGCTAATATCCAACAAAGGAAA } \\
\text { R: AGGAGCATGTTATGCTATTACCAACA }\end{array}$ & $52 / 60$ & 159 & FJ147825 & 32 & $\begin{array}{l}150- \\
900\end{array}$ & $\mathrm{P}$ \\
\hline 39 & BSSR-14 & (TTA)4 (p3) & $\begin{array}{l}\text { F: TACCCATAACTCAAGTTGGATAATTC } \\
\text { R: AATGTCTAAACCCACTGATTTAAAAG }\end{array}$ & $52 / 58$ & 240 & FJ816268 & 30.8 & 250 & + \\
\hline
\end{tabular}


Hille et al., (2002) reported that monomorphic loci observed in cross amplification could be due to null heterozygous condition. Four (GSSR-43, GSSR-96, GSSR-113 and BSSR-8) out of thirty nine SSR markers revealed polymorphic loci. Moreover non-specific amplifications were observed in seven primers, namely, GSSR-40, GSSR-81, GSSR113, GSSR-140, GSSR-141, GSSR-153 and BSSR-6, and one primer GSSR-113 was showing non-specific but polymorphic banding pattern (Table.1). Our data provide an undoubted evidence for the potential transferability of SSRs in plants more closely related to carrot like coriander, as earlier reported that closely related species share similar SSR priming sites that results in easier transferability of SSRs (Rai et al., 2013). The impact of evolutionary relationship between source and target species on SSR transfer success have been widely observed in many plant families (Rossetto et al., 2001; Arnold et al., 2002). Transferability of carrot SSR markers across coriander is achieved in this study which proves that screening of SSR markers from different species can lead to the development of SSR loci in coriander. It may further be possible to increase the success rate of cross-species SSR amplification by searching expressed sequence tags (ESTs) and heterogeneous nuclear RNA (hnRNA) libraries or database for SSRs that can be anchored in exons. Therefore, these SSR loci should be a powerful tool for coriander breeding programs and conservation genetic studies.

\section{Acknowledgment}

The senior author is thankful to the Director, NRCSS, Ajmer, Rajasthan for provide necessary facility to carry out this experiment.

\section{References}

Aldrich, P.R., Jagtap, M., Michler, C.H. and Romero-Severson, J. 2003.
Amplification of North American red oak microsatellite markers in European white oaks and Chinese chestnut. Silvae Genet., 52:176-179.

Al-Kordy, M.A., Abou El-Nasr, T.H.S. and Sherin, A.M. 2013. Assessing phenotypic and molecular variability in coriander (Coriandrum sativum L.) cultivars. J. Appl. Sci. Res., 9:38803889.

Arnold, C., Rossetto, M., McNally, J. and Henry, R.J. 2002. The application of SSRs characterized for grape (Vitis vinifera) to conservation studies in Vitaceae. Am. J. Bot., 89:22-28.

Aruna, G. and Baskaran, V. 2010. Comparative study on the levels of carotenoids lutein, zeaxanthin and bcarotene in Indian spices of nutritional and medicinal importance. Food Chemistry, 123:404-409.

Barbara, T., Palma-Silva, C., Paggi, G.M., Bered, F., Fay, M.F. and Lexer, C. 2007. Cross-species transfer of nuclear microsatellite markers: Potential and limitations. Mol. Ecol., 16:3759-3767.

Barreneche,T., Bodenes, C., Lexer, C., Trotin, J.F., Funchs, S., Streiff, R., Plomoin, C., Roussel, G., Steinkellner, H., Burg, K., Favre, J.M., Glossl, J. and Kremer, A. 1998. Agenetic linkage map of Quercus robur L. (pendunculate oak) with RAPD, SCAR, microsatellite, minisatellite, isozyme and rDNA markers. Theor. Appl. Genet., 97:10901103.

Bushar, L.M., Maliga, M. and Reinert, H.K. 2001. Cross-species amplification of Crotalus horridus microsatellites and their application in phylogenetic analysis. J. Herpetol, 35:532-537.

Butcher, P.A., Decroocq, S., Gray, Y. and Moran, G.F. 2000. Development, inheritance and cross-species amplification of microsatellite markers from Acacia mangium. Theor. Appl. 
Genet., 101:1282-1290.

Caporale, L.H. 2003. "Natural selection and the emergence of a mutation phenotype: an update of the evolutionary synthesis considering mechanisms that affect genome variation". Ann. Rev. Micro. 57:467-485.

Cavagnaro, P.F., Chung, S.M., Manin, S., Yildiz, M., Ali, A., Alessandro, M.S., Iorizzo, M., Senalik, D.A. and Simon, P.W. 2011. Microsatellite isolation and marker development in carrot - genomic distribution, linkage mapping, genetic diversity analysis and marker transferability across Apiaceae. BMC Genomics, 12:386.

Cipriani, G., Lot, G., Huang, W.G., Marrazzo, M.T., Peterlunger, E., Testolin, R. 1999. AC/GT ans AG/CT microsatellite brepeats in peach [Prunus persica (L) Barsch]: isolation, characterization and cross-species amplification in Prunus. Theor. Appl. Genet., 99:65-72.

Doyle, J.J. and Doyle, J.L. 1990. Isolation of plant DNA from fresh tissue. Focus, 12:13-15.

Glenn, T.C. and Schable, N.A. 2005. Isolating microsatellite DNA loci. Meth. Enzymol., 395:202-222.

Gupta, P.K. and Varshney, R.K. 2000. The development and use of microsatellite markers for genetic analysis and plant breeding with special emphasis on bread wheat. Euphytica, 113:163-185.

Hille, A., Janssen, I.A., Menken, S.B., Schlegel, M., Thorpe, R.S. 2002. Heterologous amplification of microsatellite markers from colubroid snakes in European Natricines (Serpentes: Natricinae). J. Hered., 93:63-66.

Jarne, P. and Lagoda, P.L. 1996. Microsatellites, from molecules to populations and back. Trends Ecol. Evol., 11: 424-429.

Kantety, R.V., Rota, M.L., Matthews, D.W. and Sorrells, M.E. 2002. Data mining for simple sequence repeats in expressed sequence tags from barley, maize, rice, sorghum and wheat. Plant Mol. Biol., 48:501-510.

Kuleung, C., Baenziger, P.S. and Dweikat, I. 2004. Transferability of SSR markers among wheat, rye, and triticale. Theor. Appl. Genet., 108:1147-1150.

Peakall, R., Gilmore, S., Keys, W., Morgante, M. and Rafalski, A. 1998. CrossSpecies amplification of soyebean (Glycine max) simple sequence repeats (SSRs) within the genus and other legume genera: Implication for the transferability of SSRs in plants. Mol. Biol. Evol., 15:1275-1287.

Qureshi, S.N., Anwar, R., Kashif, M. and Ghafoor, A. 2009. Evaluation of winter vegetables for genetic divergence and characterization of genotypes. Pak. J. Bot., 41: 1117-1126.

Rai, M.K., Phulwaria, M. and Shekhawat, N.S. 2013. Transferability of simple sequence repeat (SSR) markers developed in guava (Psidium guajava L.) to four Myrtaceae species. Mol. Biol. Rep. 40:5067-71.

Ramasamy, Y., Ghosh, M., Sumathi, R. and Gurumurthi, K. 2005. Cross-species amplification of eucalyptus SSR markers in Casuarinaceae. Acta Bot. Croat., 64:115-120.

Roder, M.S., Plaschke, J., Konig, S.U., Borner, A., Sorrells, M.E., Tanksley, S.D. and Ganal, M.W. 1995. Abundance, variability and chromosomal location of microsatellites in wheat. Mol. Gen. Genet. 246:327-333.

Rossetto, M. 2001. Sourcing of SSR markers from related plant species. In: Plant genotyping: the DNA fingerprinting of plants. Henry RJ, Wallingford, UK, CAB International pp 211-224.

Schnell, R.J., Olano, C.T., Quintanilla, W.E. 
and Meerow, A.W. 2005. Isolation and characterization of 15 microsatellite loci from mango (Mangifera indica L.) and cross-species amplification in closely related taxa. Mol. Ecol. Notes, 5:626627.

Singh, R.K., Verma, S.S., Meena, R.S. and Kumar, R. 2013. Characterization of coriander (Coriandrum sativum L.) varieties using SDS-PAGE and RAPD markers. Afr. J. Biotechnol., 12:11891195.

Steinkellner, H., Lexer, C., Turestschek, E. and Glossl, J. 1997. Conservation of (GA) $\mathrm{n}$ microsatellite loci between Quercus species. Mol. Ecol. 6:11891194.

\section{How to cite this article:}

Sharda Choudhary, Geetika Jethra, Radheshyam Sharma and Arvind Kumar Verma. 2017. Microsatellite in Coriander: A Cross Species Amplification within Apiaceae Family. Int.J.Curr.Microbiol.App.Sci. 6(5): 2714-2721. doi: https://doi.org/10.20546/ijcmas.2017.605.304 